\title{
The CEBAF separator cavity resonance control system*
}

\author{
M. Wissmann", C. Hovater, A. Guerra, T. Plawski \\ TJNAF, Newport News, VA.
}

\begin{abstract}
The CEBAF energy upgrade will increase the maximum beam energy from $6 \mathrm{GeV}$ to $12 \mathrm{GeV}$ available to the experimental halls. RF deflection cavities (separators) are used to direct the electron beam to the three halls. The resulting increase in RF separator cavity gradient and subsequent increase in RF power needed for these higher energies will require the cavities to have active resonance control. Currently, at the present 4 to $6 \mathrm{GeV}$ energies, the cavities are tuned mechanically and then stabilized with Low Conductivity Water (LCW) which is maintained at a constant temperature of $95^{\circ}$ Fahrenheit. This approach is no longer feasible and an active resonance control system that controls both water temperature and flow has been designed and built. The system uses a commercial PLC with embedded PID controls to regulate water temperature and flow to the cavities. The system allows the operator to remotely adjust temperature/flow and consequently cavity resonance for the full range of beam energies. Ultimately, closed loop control will be maintained by monitoring each cavity's reflected power. This paper describes this system.
\end{abstract}

\section{INTRODUCTION}

The Continuous Electron Beam Accelerator Facility, (CEBAF) will undergo a planned energy upgrade of the accelerator allowing the beam to reach energies of up to $12 \mathrm{GeV}$. This upgrade requires new capabilities and subsequent redesigns of the machine's RF systems. One of these systems, the RF Separators, has been upgraded to support the additional RF power requirements, but enhancements to the cavity resonance control were not done at the same time [1]. Currently, the separator cavities are tuned with local mechanical tuners at the cavities and then stabilized with low conductivity water (LCW) [2]. The tuning procedure interrupts beam operations as it requires tunnel entry and taking a network analyzer down to the cavities. The cavities are tuned 20 to $30 \mathrm{KHz}$ above the $499 \mathrm{MHz}$ cavity operating frequency to compensate for the RF heating during operation. As the beam energy has increased from $4 \mathrm{GeV}$ to $6 \mathrm{GeV}$, the increase in RF power required to kick higher energy beam has resulted in more frequent tuning of the cavities. At times, cavity VSWRs of $2: 1$ or even $3: 1$ are not uncommon, though are at least, temporarily tolerated. Another factor affecting the tuning stability of the cavities is variations in the LCW water temperature. The LCW system currently supplies constant flow $95^{\circ}+/-3^{\circ} \mathrm{F}$ water to stabilize the cavity. As other systems that use LCW (especially magnets and their power supplies) are brought on line or vary their loads, LCW supply temperature and cavity resonance are affected. The $12 \mathrm{GeV}$ upgrade will only amplify these effects and necessitates some sort of active resonance control. The system we have implemented uses two PID control loops controlling, a $208 \mathrm{VAC}, 3 \mathrm{~kW}$ heater, and a 3-way mixing valve for each cavity to control/stabilize cavity resonance (see Fig. $1)$.

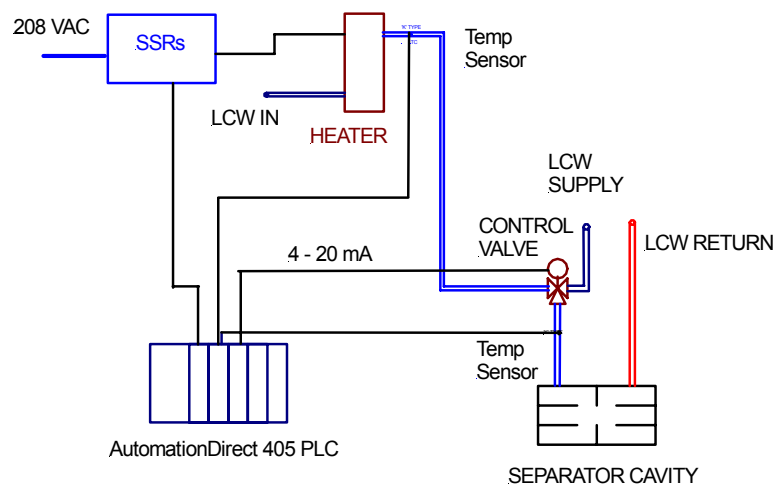

Figure 1: Cavity Resonance System

\section{THE CONTROL SYSTEM}

\section{Water System Components}

The main water system components are the heater assembly and the mixing valve. While not yet defined, the mixing valve being considered is a Honeywell VC7936 series actuator connected to a linear response, model VCZM_1600 3-way Balanced Hydronic Valve. $120^{\circ} \mathrm{F}$ water from the heater assembly will be mixed, in proportion with valve position, with $95^{\circ} \mathrm{F}$ LCW (Figure 2). These valves will be installed locally at the cavity to minimize the response time.

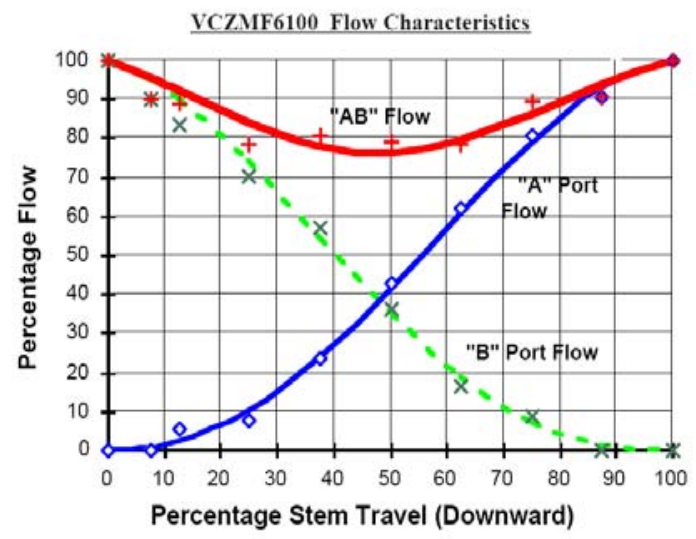

Figure 2: Typical 6100 series flow characteristics [3] 
The heater assembly is designed as a modular unit (Figure 3). They are located on the bottom portion of the control rack. All connections to the heater unit are in the rear, and polyurethane slides allow easy removal for replacement or repair (Figure 4). Each unit contains one $208 \mathrm{VAC}$, single phase, $3 \mathrm{~kW}$ heater. Each was specified to achieve $120^{\circ} \mathrm{F}$ temperature water with $95^{\circ} \mathrm{F} \mathrm{LCW}$ at a flow rate of 0.6 to $0.7 \mathrm{gpm}$. A manual throttle valve located at the heater's input facilitates setup of the desired flow rate, which ultimately determines the maximum obtainable water temperature out of the heater. Each heater's fuse, control solid state relay (SSR), and over temperature SSR are integral to its own assembly. Flow switches, set at $0.5 \mathrm{gpm}$, located in the LCW line downstream of each cavity will provide interlock

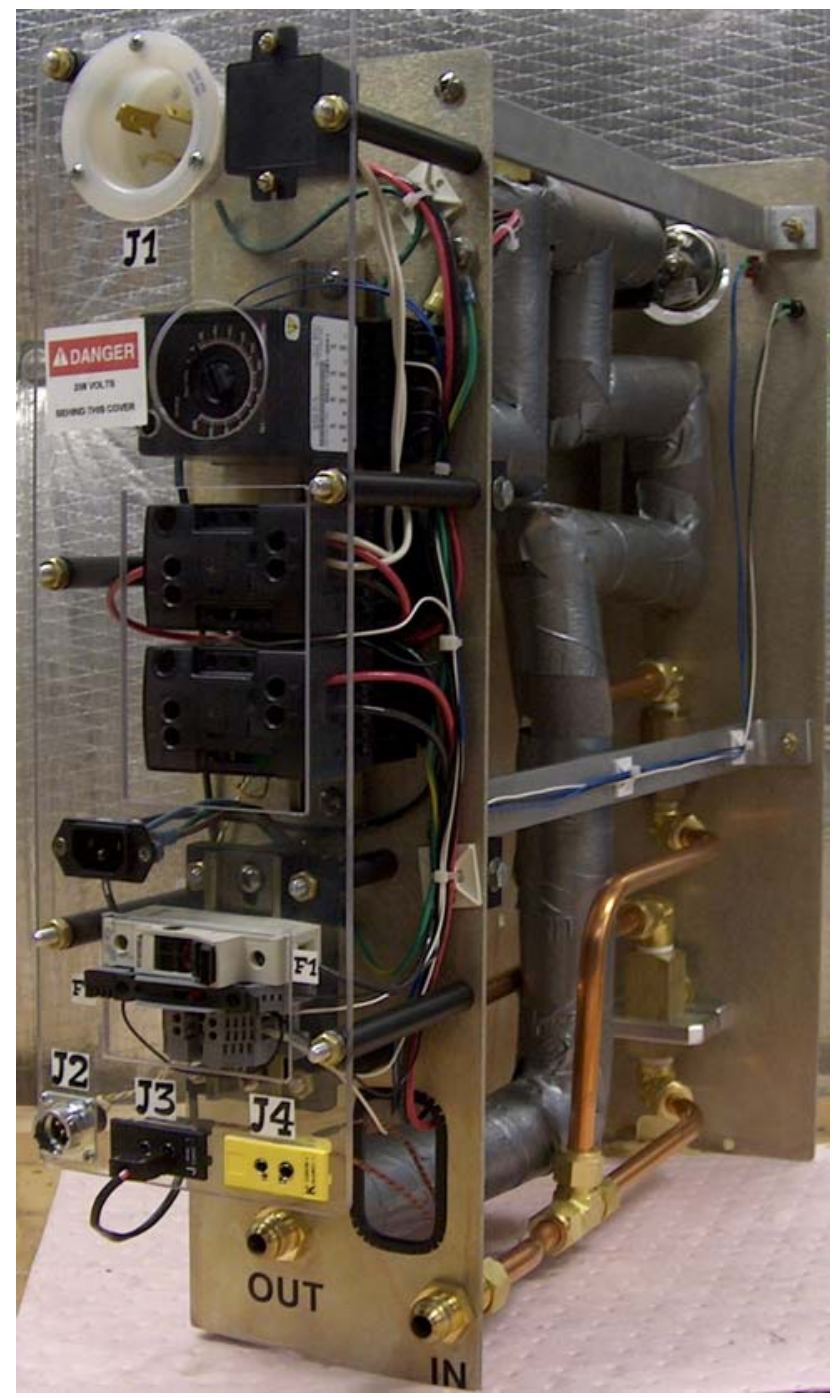

Figure 3: Rear view of a heater assembly. protection in case of leaks or loss of flow - a condition we have already experienced.

\section{The PLC}

The PLC is from AutomationDirect's DL405 family. A D4-450dc-1 CPU is installed in an 8-slot base. There are two assemblies of these in the system, locatedat opposite ends of the service building. One controls the seven Pass 1 through Pass 4 cavities, while a second controls the three cavities in Pass 5. The 8 slots make connecting the various combinations of input, output or communication modules simpler. Each PLC contains 16 independent PID loops allowing for control of 8 cavities. An additional PID loop module is available to accommodate the expansion expected for the $12 \mathrm{GeV}$ upgrade.

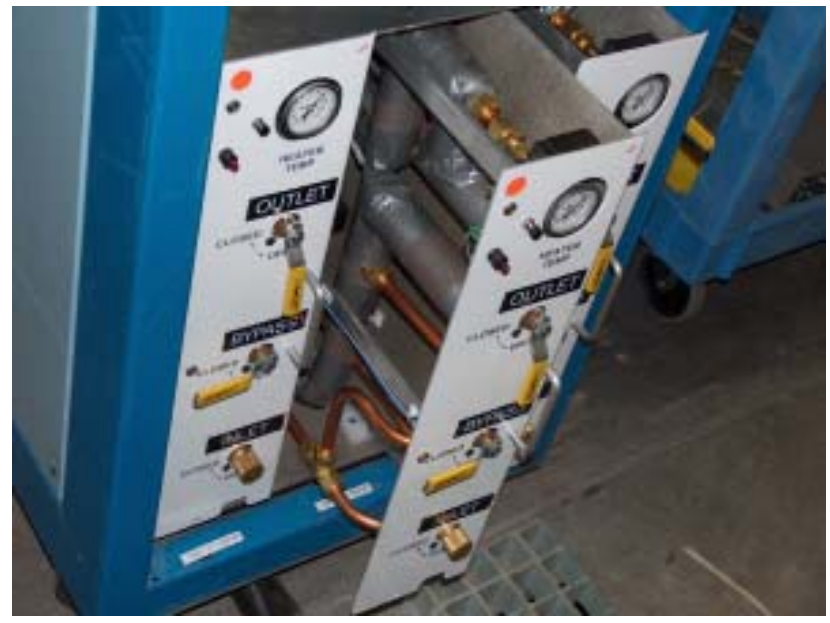

Figure 4: Heater units inserted I a rack. Heater assembly for Cavity 5-2 is shown partially removed to show the polyurethane slide.

\section{PID controls}

Sixteen PID algorithms reside in each AutomationDirect 450 PLC. Each cavity will utilize two algorithms. One controls the heater output water temperature at $120^{\circ} \mathrm{F}$, the second controls a mixing valve which maintains constant water temperature into the cavity by combining $95^{\circ} \mathrm{F} \mathrm{LCW}$ with $120^{\circ} \mathrm{F}$ heater water. By adjusting temperature, the system is essentially a remote cavity tuner. Resonance is set manually by monitoring reflected power with power meters attached to directional couplers at the output of the Inductive Output Tube (IOT) amplifier [1]. Reflected power is minimized by changing the PID loop set point. The first algorithm uses this set point to output a time-proportioning signal to vary the duty cycle of a 24 VDC PLC output. This signal turns on and off a 208 VAC solid state relay (SSR) connected in series with the heater to maintain the cavity input temperature at the set point $+/-0.5^{\circ} \mathrm{F}$. The set point is changed locally by key entry on the EZText operator interface mounted on the rack's transparent polycarbonate front panel (Figure. 5). Future enhancements will use a 0 -10 volt signal which is a function of the reflected power phase. The second loop will use this signal in a 
continuous control algorithm to send $4-20 \mathrm{~mA}$ to control the mixing valve. Communications with the PLC will eventually allow us to monitor and change any parameters remotely through CEBAF EPICS operations screens.

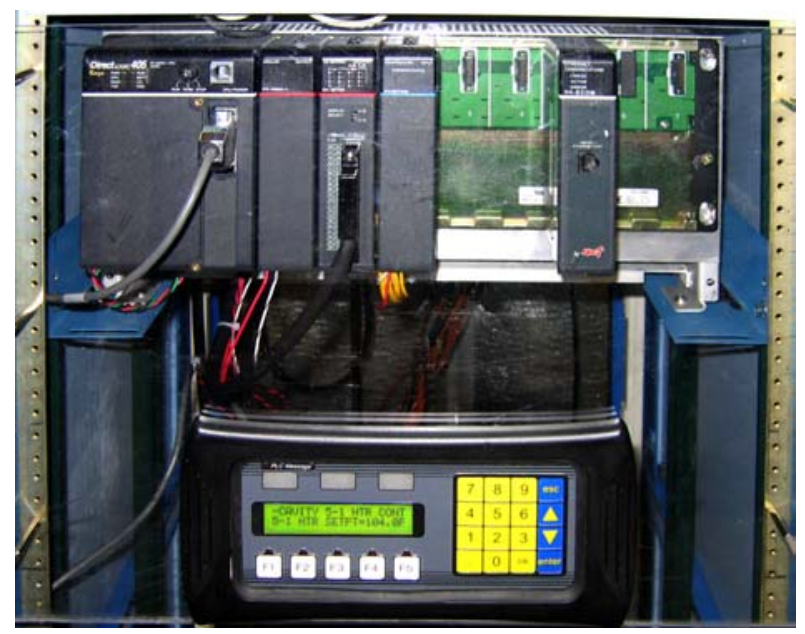

Figure 5: PLC and EZText operator interface panel mounted in the control rack.

\section{STATUS \& FUTURE PLANS}

Initial measurements of cavity frequency response as a function of temperature showed an $8 \mathrm{KHz} /{ }^{\circ} \mathrm{C}$ change (Figure 6). Frequency was measured with a Network Analyzer and temperature with a $\mathrm{K}$ type thermocouple. This measurement did not allow us to observe the effects from high power RF. Changes in RF power and water conditions produce both immediate and delayed effects on resonant frequency. First, the mode enhancing copper rods concentrate the RF fields in the center of the cavity [4]. Their location and function in the cavity along with the fact that water flows directly through them means any change in RF power or water conditions cause a quick and significant change in resonant frequency (within seconds). This effect is only partially observed in the above test. Secondly, the cavities are large and made mostly of stainless steel. These inherent characteristics cause the cavity geometry to change slowly $(10-15$ minutes $)$ and produce a delayed effect.

Recently, a partial system was installed on the Pass 5 cavities, which allowed more comprehensive testing. After allowing the cavities to soak at an elevated temperature $\left(105^{\circ} \mathrm{F}\right)$ for $>12$ hours, a tunnel entry was made to set the resonant frequency of the cavities $\sim 40$ $\mathrm{KHz}$ above the desired $499 \mathrm{MHz}$. RF was then applied while monitoring forward and reflected powers. Reflected power was minimized by lowering the heater output temperature as RF power was increased. This proved effective and the system is currently operating in this state.

The above-mentioned mixing valve is still in testing as of this writing. Other mixing valves tested have not yielded linear mixing characteristics as promised. This has been a problem and has delayed that portion of the system. We plan to install and incorporate the mixing valve into the system in September of 2005.

Ultimately we plan to use an automated resonance control scheme which monitors individual cavity reflected power. It was originally thought that a custom RF receiver would be used to accomplish this task. However, as part of the accelerator availability improvements we are developing new RF controls for the $499 \mathrm{MHz}$ RF systems [5]. We intend to use the four receiver channels of this system to measure cavity reflected power and phase. This signal would then be sent to the PLC and used as the process variable in the PID algorithm

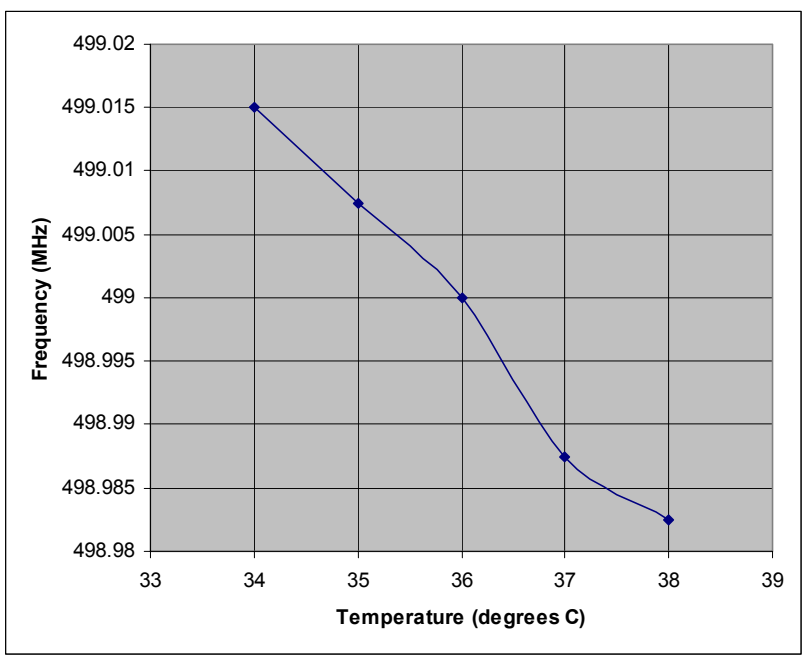

Figure 6: Temperature vs. Frequency Graph of CEBAF Pass 5-3 Separator Cavity. Data taken in March 2003.

\section{REFERENCES}

[1] C. Hovater, et. al, "THE CEBAF RF SEPARATOR SYSTEM UPGRADE”, LINAC 2004 conference proceedings, Lubeck, Germany, August 2004

[2] C. Hovater, et al, "The CEBAF RF Separator System", LINAC 1996 conference proceedings, Geneva, Switzerland, August 1996

[3] www.honeywell.ca/water-controls/

[4] A. Krycuk, et al, "Construction of the CEBAF RF Separator”, 1993 Particle Accelerator Conference, May 1993.

[5] C. Hovater et al, "A New RF system for the CEBAF Normal Conducting Cavities" LINAC 2004 conference proceedings, Lubeck, Germany August 2004 